\title{
Open
}

\section{TLR1-induced chemokine production is critical for mucosal immunity against Yersinia enterocolitica}

\author{
Y Sugiura $^{1}$, K Kamdar ${ }^{2}$, S Khakpour ${ }^{3}$, G Young ${ }^{4}$, WJ Karpus ${ }^{5}$ and R William DePaolo ${ }^{2}$
}

Our gastrointestinal tract is a portal of entry for a number of bacteria and viruses. Thus, this tissue must develop ways to induce antigen-specific T cell and antibody responses quickly. Intestinal epithelial cells are a central player in barrier function and also in communicating signals from invading pathogens to the underlying immune tissue. Here we demonstrate that activation of Toll-like receptor 1 (TLR1) in the epithelium leads to the upregulation of the chemokine CCL20 during oral infection with Yersinia enterocolitica. Further, both neutralization of CCL20 using polyclonal antibody treatment and deletion of TLR1 resulted in a defect in CCR6 + dendritic cells (DCs), which produce innate cytokines that help to induce anti-Yersinia-specific T helper $17\left(T_{H} 17\right)$ cells and IgA production. These data demonstrate a novel role for TLR1 signaling in the intestinal epithelium and demonstrate that together TLR1 and CCL20 are critical mediators of $T_{H} 17$ immunity through the activation and recruitment of DCs.

\section{INTRODUCTION}

The mucosal immune system must balance tolerogenic responses against food and commensal microbiota while preserving the ability to mount protective inflammatory responses against invading pathogens. This delicate balance is maintained through the coordinated signals and interactions of a variety of specialized cells. Besides providing an important barrier between invading pathogens and the underlying tissue, intestinal epithelial cells (IEC) can interact with both the bacteria via secretion of antibacterial peptides, ${ }^{1}$ and the immune system through the production of cytokines and chemokines. Within the intestinal epithelium is a further specialized tissue called follicular-associated epithelium (FAE), which is situated over lymphoid follicles called Peyer's patches (PPs). M cells within the FAE directly sample luminal antigens by macro-pinocytosis, permitting the delivery of antigen into the PPs where they can be taken up by dendritic cells (DC) and presented to naive T cells. A number of enteric pathogens such as Salmonella typhimurium $^{2,3}$ and Y. enterocolitica ${ }^{4}$ have developed strategies to target the FAE and $M$ cells in order to penetrate the epithelium and cause disease. The epithelium is able to respond to invading bacteria by the activation of pattern recognition receptors such as NOD-like and Toll-like (TLR) receptors.
These receptors recognize conserved pathogen-associated molecular patterns such as peptidoglycan, flagellin, or lipoproteins. TLR activation induces an innate immune response, which in part, will direct the migration of DCs to the site of the infection. DCs have the significant task of integrating the signals derived from pathogens ${ }^{5}$ but also from the tissue microenvironment. ${ }^{6,7}$ The integration of these signals is crucial to the induction of appropriate CD4 $\mathrm{T}$ helper $\left(\mathrm{T}_{\mathrm{H}}\right)$ responses that can eliminate the pathogen while limiting damage to the infected tissue. Hence, the recruitment and activation of DCs by IEC is a critical step in a fundamental immune cascade leading to protection against mucosal pathogens.

The trafficking of DCs to the site of inflammation is dependent upon the secretion of chemokines. ${ }^{8}$ The chemokine CCL20 (also known as Macrophage Inhibitory Protein-3a) is constitutively expressed in the gastrointestinal tract, ${ }^{9}$ however stimulation of IEC by inflammatory cytokines, ${ }^{10}$ bacteria such as Salmonella Typhimurium ${ }^{11}$ or the TLR5 ligand, flagellin, ${ }^{12}$ can also stimulate production of CCL20. CCL20 binds to CCR6, ${ }^{13,14}$ a G-protein-coupled receptor expressed on the surface of leukocytes, including lymphocytes ${ }^{15,16}$ and DCs. ${ }^{17}$

CCL20 and its receptor CCR6 have been shown to be important for protection against a number of mucosal infections, such

\footnotetext{
${ }^{1}$ Department of Biomedical Sciences, Midwestern University, Downers Grove, Illinois, USA. ²Department of Molecular Microbiology and Immunology, University of Southern California, Keck School of Medicine, Los Angeles, California, USA. ${ }^{3}$ Department of Medicine, University of Chicago, Chicago, Illinois, USA. ${ }^{4}$ Department of Food Science and Technology, University of California Davis, Davis, California, USA and ${ }^{5}$ Department of Pathology, Northwestern University, Feinberg School of Medicine, Chicago, Illinois, USA. Correspondence: RW DePaolo (depaolo@usc.edu)
}

Received 4 October 2012; accepted 7 January 2013; published online 27 February 2013. doi:10.1038/mi.2013.5 
as respiratory syncytial virus, ${ }^{18}$ Helicobacter pylori, ${ }^{19}$ and $S$. Typhimurium. ${ }^{20}$ Pathological examination of colonic biopsies revealed CCL20 is elevated in patients with inflammatory bowel disease (IBD) ${ }^{21}$ and Genome Wide Association Studies (GWAS) revealed CCL20 is a susceptibility gene for Crohn's disease. 22 These data indicate that CCL20 and CCR6 have important roles in protection against mucosal infection, but are also implicated in the development of mucosal inflammatory disease.

Previously we have demonstrated that protective $\mathrm{T}_{\mathrm{H}} 17$ immunity against oral $Y$. enterocolitica infection requires signaling via TLR $1 .{ }^{23}$ In the absence of TLR1, there is also a deficiency in the accumulation of DCs in the gut-associated lymphoid tissue after Y. enterocolitica infection. In the present study, we identify a novel role for TLR1 signaling in the intestinal epithelium leading to the initiation of DC migration and ultimately the generation of protective $\mathrm{T}_{\mathrm{H}} 17$ and $\operatorname{IgA}$ immunity against enteric $Y$. enterocolitica infection.

\section{RESULTS}

CCL20 induction is independent of invasion and type III secretion but dependent upon TLR1 stimulation

Our previous study demonstrated that TLR1 ${ }^{-1-}$ mice were unable to effectively mount a protective mucosal $\mathrm{T}_{\mathrm{H}} 17$ response during mucosal infection by Y. enterocolitica. This was due to a defect in the activation of TLR1-deficient DCs through direct contact with $Y$. enterocolitica, resulting in decreased interleukin (IL)- 6 and ultimately decreased $\mathrm{T}_{\mathrm{H}} 17$ priming. ${ }^{23}$ Interestingly, TLR $1^{-1-}$ mice also had fewer DCs in the mesenteric lymph node (MLN) 3 days following infection. IECs come in contact with bacteria during infection and secrete CCL20 to attract CCR6 + DC to the site of infection. ${ }^{9}$ After activation, CCR6 + $\mathrm{CD} 11 \mathrm{c}+$ cells downregulate CCR6 and traffic to draining lymph nodes, where they present antigens to naive $\mathrm{T}$ cells. We hypothesized that the reduction of DCs observed in the MLN of TLR1 ${ }^{-1-}$ mice may be due to a defect in recruitment of DCs to the site of infection. To address the mechanism of DC trafficking in TLR1-deficient mice, CCL20 expression was examined in mucosal tissues $72 \mathrm{~h}$ after oral infection with Y. enterocolitica. Y. enterocolitica-induced CCL20 expression was reduced in the IECs and PPs of TLR1 ${ }^{-1-}$ mice compared with wild-type littermate controls by both protein (Figure 1a) and messenger RNA (mRNA; Figure 1b). CCL20 expression was not induced in either the lamina propria (LP) or MLN of littermate controls or TLR1-deficient mice (Figure 1a, b). Interestingly, the absence of TLR6, which also forms a heterodimer with TLR2, ${ }^{24,25}$ showed no effect on CCL20 production (data not shown), suggesting a specific role for TLR1 in the IEC for CCL20 expression. To show directly that CCL20 was being produced by IECs in a TLR1-dependent manner, IECs and lamina propria immune cells (LPCs) were isolated using expression of CD13 and CD45. IECs (CD13+ CD45-) and LPCs (CD13-CD45+) were purified from the ileum of the small intestine from TLR1 $1^{-1-}$ and wild-type littermate control mice. CCL20 mRNA was measured after stimulation with TLR ligands or Y. enterocolitica. CCL20 transcript was induced by TLR2/1 ligand and Y. enterocolitica stimulation of IEC (Figure 1c, left) but not LPC (Figure 1c, right). These data indicate a TLR1 dependence for CCL20 production in IECs but not LPCs.

A common single-nucleotide polymorphism (SNP) occurring in the transmembrane domain of TLR1 abrogates cell surface expression and signaling ${ }^{26}$ and is present in at least $20 \%$ of the human population. ${ }^{27}$ Using a human IEC line that does not express TLR1 ${ }^{28}$ (data not shown), we examined the ability of the wild-type TLR1 allele (I602I) and the mutant TLR1 allele (I602S) to induce CCL20 after transfection. Both the TLR2/1 ligand Pam3Cysk4 and Y. enterocolitica lysate induced significant levels of CCL20 in IEC transfected with the wild-type allele (Figure 1d, black bars). However, CCL20 expression was not induced if the IEC were transfected with the TLR1 I602S (Figure 1d, white bars). Notably, Y. enterocolitica lysate was still able to induce CCL20 in the Caco-2 cells transfected with the vector control, which may be due to stimulation of TLR5 by flagellin ${ }^{11,29}$ or other TLR agonists. ${ }^{30,31}$ These data demonstrate that epithelial expression of TLR1 is essential for the induction of CCL20 after oral Y. enterocolitica infection and suggests that individuals with the I602S SNP in TLR1 may be unable to induce CCL20 expression during mucosal infection by $Y$. enterocolitica.

$Y$. enterocolitica harbors genes required for cellular invasion, such as invA and $y a d A$, or genes encoding two different type III secretion systems (T3SSs; $y s a$ and $y s c$ ). These T3SSs inject effector proteins, called Ysps (Yersinia-secreted proteins) and Yops (Yersinia outer proteins), into target cells. ${ }^{32,33}$ These effectors have multiple and diverse functions such as impairing cell signaling, inhibiting actin re-arrangement, or inhibiting nuclear factor- $\kappa \mathrm{B}$ activation. ${ }^{34}$ Yops have also been shown to activate intracellular immune pathways. ${ }^{35}$ A specific effector protein of the Ysc T3SS, LcrV, has been shown to be essential for immune-evasion via IL-10 production ${ }^{36}$ and is also critical for the formation of the Ysc T3SS needle. ${ }^{37}$ In order to determine whether invasion or the T3SS are important for the induction of CCL20, Caco-2 cells were transfected with wild-type TLR1 (I602I) and the production of CCL20 was measured after stimulation with $Y$. enterocolitica or deletion mutants. The supernatants were collected and used in a chemotaxis assay with differentiated human $\mathrm{CD} 34+\mathrm{DC}$ to determine whether chemo-attraction occurred in a CCL20-dependent manner. Supernatants from TLR1 I602I-expressing IECs stimulated with wild-type $Y$. enterocolitica (8081) were able to induce the migration of cells similar to that of recombinant human CCL20 (rCCL20). However, the addition of neutralizing CCL20 antibody to the supernatant almost completely inhibited the migration of DCs, confirming a specific role for CCL20 in this migration (Figure 2a). Supernatants from IECs expressing wild-type TLR1 induced a CCL20-dependent migration that proved to be independent of invasion, type III secretion, and $\mathrm{LcrV}$, as each deletion mutant was able to stimulate CCL20 expression equal to wild-type Y. enterocolitica (Figure 2a). TLR5 signaling by flagellin has been shown to induce CCL20 expression $^{11,29}$ and may account for the further reduction seen in I602S-transfected Caco-2 cells treated with anti-CCL20. To 

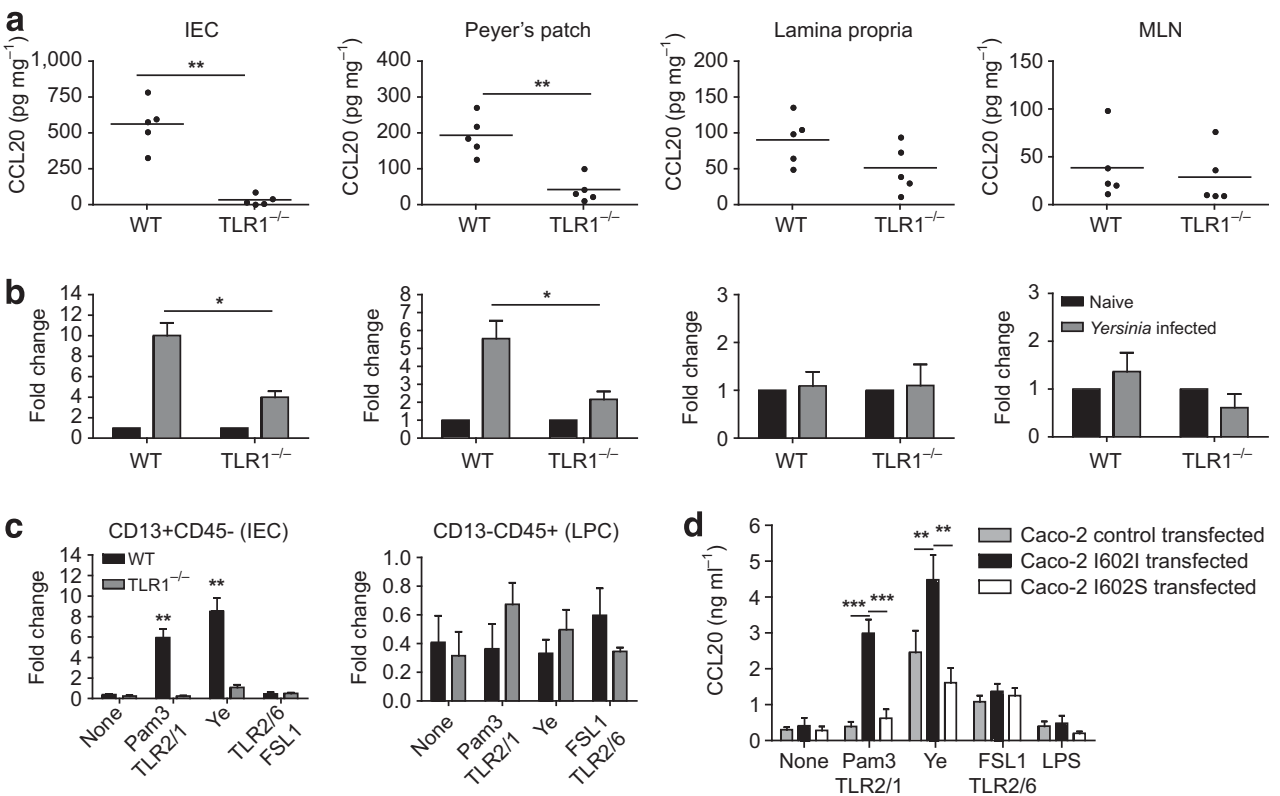

Figure 1 CCL20 is secreted in a Toll-like receptor 1 (TLR1)-dependent manner from intestinal cells. Levels of CCL20 protein (a) and messenger RNA (mRNA; b) from various mucosal tissues 3 days after oral $Y$. enterocolitica infection. Data are pooled from two independent experiments ( $n=4-6$ mice per group). (c) Levels of CCL20 mRNA in intestinal epithelial cells (IECs; CD13 + CD45-) and lamina propria immune cells (LPCs; CD13-CD45 + ) sorted from wild-type (WT) and TLR1 ${ }^{-1-}$ mice. Purified cells were stimulated with TLR2/1 ligand, TLR2/6 ligand, and Y. enterocolitica (Ye). Data are the average from three to four individual mice. (d) Level of CCL20 from Caco-2 cells transfected with control vector (control), WT TLR1 (1602I), or TLR1 containing a single-nucleotide polymorphism (I602S) $18 \mathrm{~h}$ after stimulation with TLR ligands or $Y$. enterocolitica lysate (Ye). Data are pooled from three independent experiments. $(n=6)$. ${ }^{\star} P<0.05$, ${ }^{\star \star} P<0.01,{ }^{\star \star \star} P<0.001$. Student's unpaired $t$-test. MLN, mesenteric lymph node.

determine whether invasion or type III secretion was necessary for the in vivo expression of CCL20, we isolated IECs from TLR1 ${ }^{-1-}$ or littermate control mice infected with Y. enterocolitica (8081) or the deletion mutants. CCL20 was equally expressed in the IEC from wild-type $Y$. enterocolitica as well as the $\Delta l c r V, \Delta y s c$, and $\Delta i n v A$ mutants (Figure $2 \mathbf{b}$ ). This was not due to the attenuation of the strains as increasing the dose by 10 - and 100-fold still had no effect on CCL20 production (data not shown). In accordance with our data in Figure 1a, TLR1deficient mice had a defect in CCL20 production from IEC and this was not altered by infection with the various mutants (Figure 2b). These data demonstrate that while invasion and type III secretion effector proteins do not contribute to the production of CCL20, TLR1 is critical for its induction.

\section{CCL20 is important for survival and clearance of $Y$. enterocolitica}

Stimulation of IECs and FAE by bacteria has been shown to induce the secretion of CCL20 ${ }^{10,38}$ and attract CCR6 + cells. $^{11}$ This interaction has been shown to be important for the generation of pathogen-specific T cells. ${ }^{20}$ Previously, we have shown that the absence of TLR1 signaling increases the mortality and bacterial burden after oral infection by Y. enterocolitica. ${ }^{23}$ Here, we wanted to investigate whether the induction of CCL20 would similarly impact the pathogenesis of mucosal Y. enterocolitica infection. Using a polyclonal neutralizing antibody against CCL20, ${ }^{18}$ we treated wild-type mice every other day after oral infection with $Y$. enterocolitica. Compared with control-treated mice, mice receiving the neutralizing antibody against CCL20 had a more severe disease phenotype, as evidenced by a higher mortality (Figure 3a) and a 10-fold higher bacterial burden in the MLN 3 days following oral infection (Figure 3b).

\section{TLR1 signaling and CCL20 are critical for the recruitment of DCs during oral $Y$. enterocolitica infection}

CCR6 + DCs have been shown to have an important role in the defense against mucosal Salmonella infection. ${ }^{20}$ These DCs are rapidly recruited to the PPs, where they are activated to prime anti-Salmonella $\mathrm{T}$ cells. ${ }^{20}$ To determine whether CCR6 + cells also have a role in $Y$. enterocolitica infection, we examined the PPs for the accumulation of CCR6 + CD11c + cells $48 \mathrm{~h}$ after mucosal Y. enterocolitica infection in TLR1-deficient mice and mice treated with neutralizing antibody against CCL20. Untreated wild-type mice or those receiving isotype control antibody developed an increase in CCR6 + CD11c + cells after Yersinia infection (Figure 4a). Consistent with a role for TLR1 in CCL20 induction, mice deficient for TLR1 demonstrated a significant reduction in the frequency (Figure $4 \mathbf{b}$ ) and total number (Figure 4c) of CCR6-expressing DCs during infection. Importantly, the defect in CCR6 + DC observed in TLR1deficient mice looked remarkably similar to the defect observed in wild-type mice treated with neutralizing anti-CCL20 antibodies (Figure 4a-c). Analysis of CCR6 expression on $\mathrm{B}$ and CD4 T cells in the LP from anti-CCL20-treated or TLR $1^{-1-}$ mice revealed no differences (data not shown). This may be due to increases in other inflammatory chemokines induced during infection that recruit adaptive immune cells. To confirm the importance of TLR1 signaling in the IEC for CCL20 production and the recruitment of CCR6 + DC during 
Y. enterocolitica infection bone-marrow chimera's were performed. Lethally irradiated TLR $1^{-1-}$ mice reconstituted with bone marrow from wild-type littermate control mice maintain the ability to signal via TLR1 in the immune cells, but lack the ability to signal through TLR1 in the IEC. These mice were unable to produce CCL20 after $Y$. enterocolitica infection (data not shown) and had a significant decrease in the recruitment of CCR6 + DC compared with wild-type mice
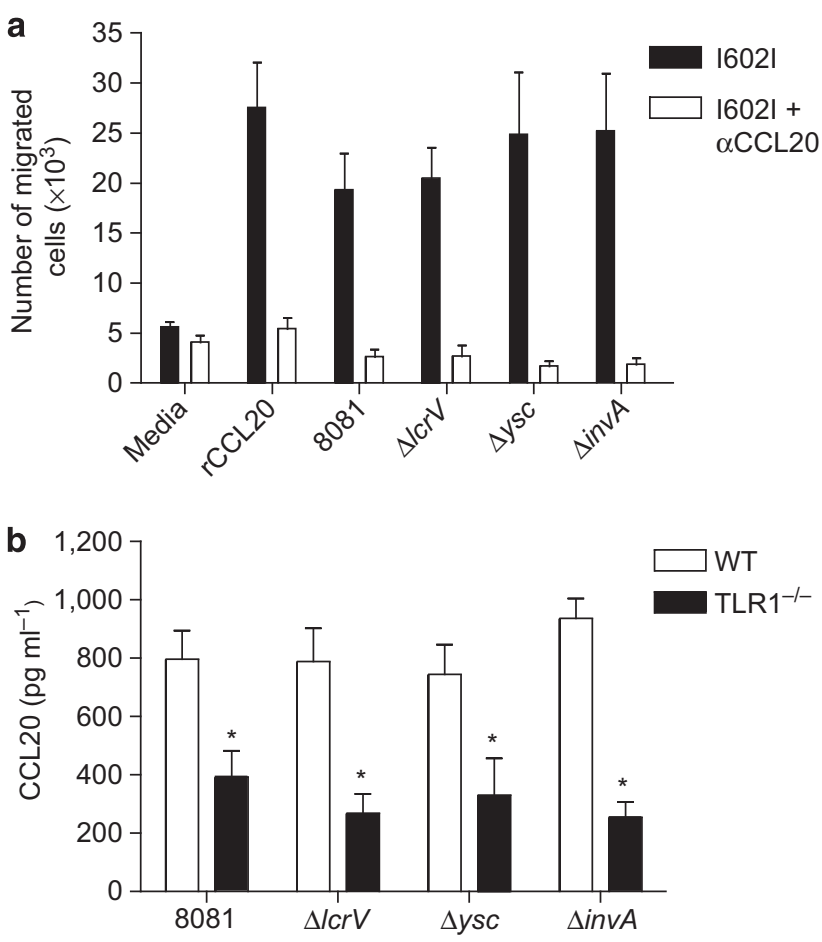

Figure 2 CCL20 production is dependent upon Toll-like receptor 1 (TLR1) signaling and not invasion or type III secretion system (T3SS). (a) Number of migrated cells toward supernatants collected from Caco-2 epithelial cells transfected with wild-type (WT) TLR1 (I602I) and stimulated with wild-type $Y$. enterocolitica (8081), $Y$. enterocolitica lacking LcrV $(\Delta / c r V)$, T3SS $(\Delta y s c)$, or invasin $(\Delta i n v A)$. Recombinant human CCL20 (rCCL20) and neutralization of CCL20 (aCCL20) were used as controls. Data are the mean \pm s.e.m. of pooled from three independent experiments. ${ }^{*} P<0.01,{ }^{* *} P<0.001$ (Student's unpaired $t$-test). (b) In vivo production of CCL20 from IECs of TLR1 ${ }^{-1-}$ or wild-type littermate control mice infected with $1 \times 10^{6} \mathrm{Y}$. enterocolitica or mutants after $72 \mathrm{~h}$. Data are the mean \pm s.e.m. of six individual mice pooled from two independent experiments. ${ }^{\star} P<0.01,{ }^{* *} P<0.001$ (Student's unpaired $t$-test). reconstituted with TLR1 ${ }^{-1-}$ bone marrow cells (Figure 4d). Overall, these data demonstrate that TLR1 signaling in the epithelium of the small intestine contributes to the recruitment of CCR6 + DC during infection by Y. enterocolitica.

\section{The absence of CCL2O results in defective innate cytokine} production, $\mathrm{T}_{\mathrm{H}} 17$ responses, and anti-Yersinia $\operatorname{IgA}$

The molecular signals that drive anti-bacterial $\mathrm{T}_{\mathrm{H}} 17$ and mucosal IgA responses are largely unknown. Even more enigmatic is the role played by the intestinal epithelium in the development of $\mathrm{T}_{\mathrm{H}} 17$ and IgA immunity. Previously, we have shown that TLR1 deficiency results in a reduction of IL- 6 and IL-23, which impacts the development of $\mathrm{T}_{\mathrm{H}} 17$ cells and ultimately IgA production. However, the observed decrease in $\mathrm{T}_{\mathrm{H}} 17$ polarizing innate cytokines, such as IL-6, in TLR1-deficient mice may also be due to initial defects in the recruitment of DCs to the site of the infection.

In order to demonstrate that CCL20 expression has an impact on $\mathrm{T}_{\mathrm{H}} 17$ responses, we examined the PP of anti-CCL20treated or TLR $1^{-1-}$ mice 3 days after oral infection with Y. enterocolitica for cytokines important in the induction and maintenance of $\mathrm{T}_{\mathrm{H}} 17$ cells. TLR1-deficient mice and mice treated with anti-CCL20 had significantly less IL-6 and IL-23 levels, but similar levels of IL-10, when compared with littermate control-treated mice (Figure 5a). In addition, we observed a significant decrease in Yersinia-specific $\mathrm{T}_{\mathrm{H}} 17$ cells in the LP 7 days following infection in both TLR1-deficient and anti-CCL20-treated mice (Figure 5b), consistent with the hypothesis that the absence of CCL20 and CCR6 + DCs would lead to an inefficient priming of $\mathrm{T}_{\mathrm{H}} 17$ cells. Of note, levels of IL-17 are more reduced in TLR $1^{-1-}$ mice compared with antiCCL20-treated mice. This is likely due to the fact that in the absence of TLR1 there is both a reduction in the trafficking of CCR6 + DC that contribute to $\mathrm{T}_{\mathrm{H}} 17$ priming and loss of IL-6 and IL-23 production due to lack of direct TLR1 signaling on MLN DC. However, anti-CCL20 treatment reduces the trafficking but disseminated $Y$. enterocolitica would still be able to stimulate IL- 6 and IL-23 from DC in the MLN. Our work ${ }^{23}$ and others ${ }^{39}$ have shown that IL-17 is important for the induction of mucosal IgA, which is important for the protection against mucosal pathogenic infections. We evaluated Yersiniaspecific fecal IgA in our anti-CCL20-treated mice and found a
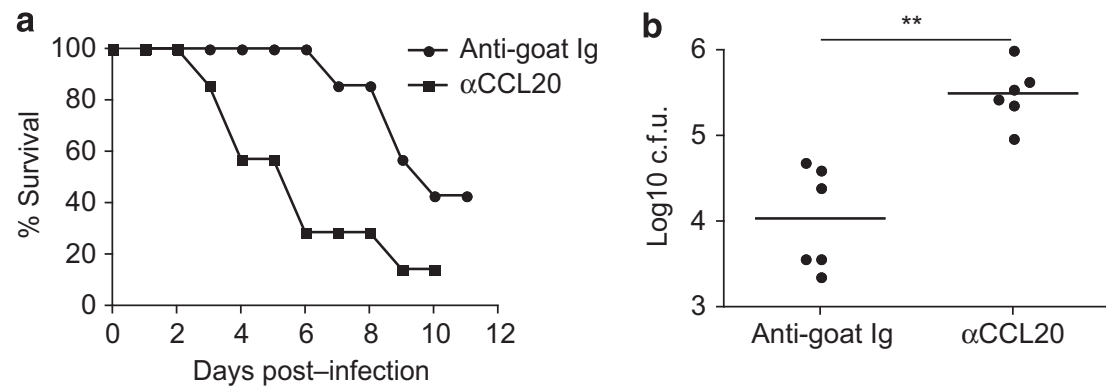

Figure $3 \mathrm{CCL} 20$ is important for survival and clearance of $Y$. enterocolitica. (a) Survival of mice fed $1 \times 10^{5}$ c.f.u. (colony-forming unit) $Y$. enterocolitica and treated with anti-CCL2 or anti-IgG polyclonal serum every other day for 10 days. $N=10$ mice per group; ${ }^{\star} P=0.0132$ (Wilcoxon Log-Rank test). (b) Bacterial burden in the mesenteric lymph node 3 days post infection. Data are pooled from two independent experiments ( $n=5$ mice per group). ${ }^{\star \star} P<0.01$ (Student's paired $t$-test). 

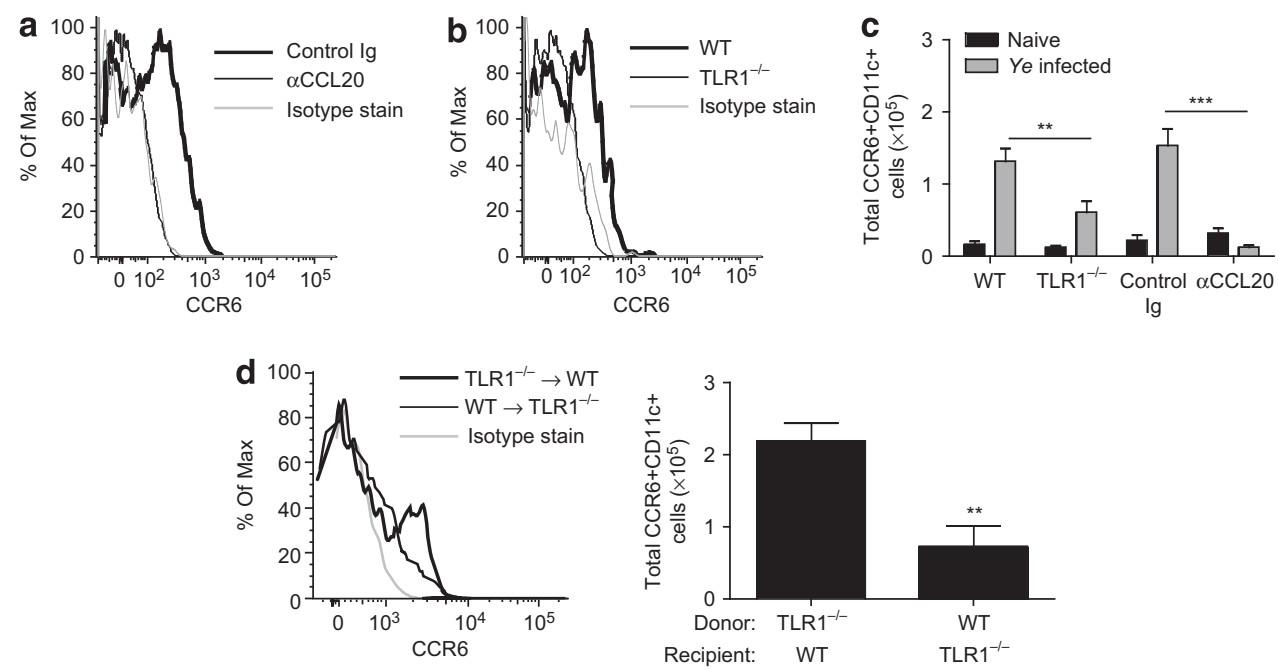

Figure 4 Toll-like receptor 1 (TLR1) and CCL20 are important for recruitment of CCR6 + CD11c + cells to the Peyer's patch (PP) during oral $Y$. enterocolitica infection. Flow cytometric analysis of dendritic cell (DC) population in PP of anti-CCL20-treated (a) and TLR1 ${ }^{-}{ }_{-}$(b) mice. Data are representative of five individual mice pooled from two experiments. (c) Total cell number of CCR6 + CD11c + cells. (d) Bone marrow chimera's were performed by reconstituting TLR $1^{-1-}$ mice with wild-type (WT) hematopoietic cells (WT $\rightarrow$ TLR1 $1^{-l-}$ ) and by reconstituting WT mice with TLR1 $^{-1-}$ hematopoietic cells (TLR1 ${ }^{-1} \rightarrow$ WT). CCR6 + DC in the PP of the bone marrow chimera's were identified by flow cytometric analysis (left) and total cell count (right) 3 days after infection with Y. enterocolitica. Data are pooled from two independent experiments ( $n=3-6$ mice per group). ${ }^{\star} P<0.05,{ }^{* \star} P<0.01,{ }^{* \star} P<0.001$ (Student's unpaired $t$-test).

significant reduction compared with control-treated mice (Figure 5c).

As CCL20 expression was important for $\mathrm{T}_{\mathrm{H}} 17$ and $\operatorname{IgA}$ production, we hypothesized that the CCR6 + DC are the cells producing IL-6 and IL-23. As a result of this elevated innate cytokine production, CCR6 + DCs would be able to induce more $\mathrm{T}_{\mathrm{H}} 17$ cells. To test this theory, CCR6 $+\mathrm{CD} 11 \mathrm{c}+$ cells were sorted from the PP of infected wild-type mice, and quantitative reverse transcription (RT)-PCR was performed to examine whether these cells express more IL- 6 and IL- 23 when compared with naive mice. As anticipated, the expression of both IL- 6 and IL-23 were significantly upregulated in the CCR6 + DC (Figure 5d). Further, CCR6 + DC stimulated $\mathrm{T}_{\mathrm{H}} 17$ cell induction as evidenced by IL-17 secretion (Figure 5e, left) and induction of the transcription factor rorgt (Figure 5e, right). Finally, to demonstrate that the IL- 6 and IL-23 produced by the CCR6 + DCs were responsible for $\mathrm{T}_{\mathrm{H}} 17$ cell induction neutralizing antibodies against IL-6, IL-23, or both were added to the cultures. IL-17 production in cultures containing CCR6 + DC was significantly reduced in the presence of anti-IL-6 or anti-IL-23 and there was an additive effect when both neutralizing antibodies were added to the culture (Figure 5f). These data suggest that the recruitment of CCR6 + DCs during infection is critical for the proper production of innate cytokines that can polarize $\mathrm{T}_{\mathrm{H}} 17$ responses and promote microbial clearance.

\section{DISCUSSION}

In this study, we demonstrate that TLR1 signaling is critical for the production of CCL20 by the intestinal epithelium during infection by $Y$. enterocolitica. Importantly, these data confirm and extend observations made by our group, which revealed a dual role of TLR1 in generating mucosal $\mathrm{T}_{\mathrm{H}} 17$ and IgA responses during mucosal infection. We identified that TLR1 was important for the induction of IL-23 and IL- 6 by DCs encountering the pathogen directly, and we also showed that in the absence of TLR1, there was a defect in the number of DCs in the MLN. ${ }^{23}$ The present study reconciles these findings by illustrating that TLR1 signaling in IEC induces CCL20. In the absence of TLR1 or CCL20, there is a defect in the recruitment of CCR6 + DC to the PPs. This population of DC is particularly important because they produce IL-6 and IL-23, and promote anti-Yersinia $\mathrm{T}_{\mathrm{H}} 17$ responses in the LP.

$Y$. enterocolitica has developed a number of virulence strategies to avoid or inhibit the immune system. The expression of invasin and YadA allow the attachment to and uptake by $\mathrm{M}$ cells of the FAE. ${ }^{4,40} Y$. enterocolitica also uses T3SS to inject effector proteins (Ysps and Yops) into host target cells. These effectors can affect a wide variety of cellular functions, such as apoptosis, nuclear factor- $\kappa \mathrm{B}$ activation, and phagocytosis. ${ }^{34}$ One effector protein secreted by the Ysc T3SS is called LcrV. LcrV is important for the injection needle, ${ }^{37}$ as well as a potent inducer of IL-10 via signaling through TLR2/6 heterodimers. ${ }^{36}$ Using mutants for invasion and type III secretion, we found that CCL20 levels were not dependent upon these virulence factors. Importantly, infection of TLR1deficient mice with the mutant Yersinia still resulted in decreased CCL20 production, and thus the induction is likely due to lipoproteins on the surface of Yersinia contacting surface expressed TLR1 on IEC, however, the identity of the particular agonist is unknown but is currently under investigation in our laboratory. The ability to recruit DC and activate adaptive immunity using TLR1 signaling even in the context of these potent virulence factors is crucial for protective immunity by the host. 

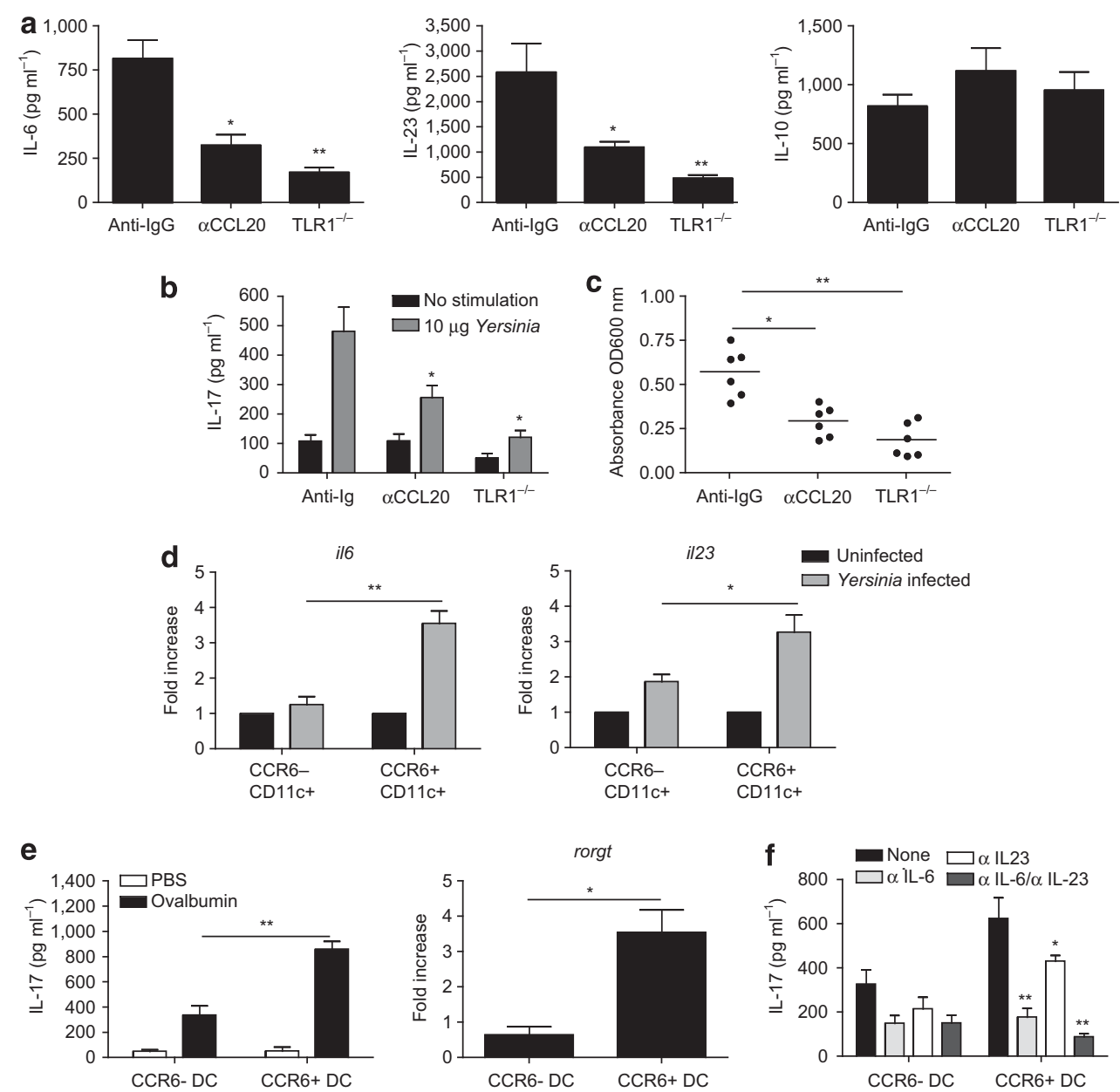

Figure 5 Absence of CCL20 impacts interleukin (IL)-17 priming and IgA secretion. (a) Levels of IL-23, IL-6, and IL-10 in the Peyer's patch of $\mathrm{TLR}^{-1}{ }^{-}$or anti-CCL20-treated mice infected orally with $Y$. enterocolitica. Data are the mean \pm s.e.m. pooled from two independent experiments $(n=6)$. (b) Levels of IL-17 from isolated LP CD4 T cells re-stimulated with irradiated antigen-presenting cell and Y. enterocolitica lysate. Data are the mean \pm s.e.m. pooled from two independent experiments $(n=6)$. (c) Fecal anti-Yersinia IgA levels from mice collected at day 14 post infection. Data are pooled from two independent experiments ( $n=6$ mice per group). (d) Messenger RNA (mRNA) levels of IL-6 and IL-23 from CCR6 + and CCR6$\mathrm{CD} 11 \mathrm{c}+$ sorted Peyer's patch cells 3 days post infection. Fold induction is compared with uninfected controls. Data are pooled from two independent experiments ( $n=5-6$ mice per group). (e) Purified CCR6 + and CCR6- dendritic cells (DCs) were sorted from wild-type mice infected with Y. enterocolitica. The purified DCs were co-cultured with CD4 + ovalbumin-specific T cells (OT2) and ovalbumin. Supernatants were analyzed after 3 days for levels of IL-17 (left). CD4 T cells were sorted from the co-cultures and mRNA level of the transcription factor for rorgt was determined. (f) Levels of IL-17 in co-cultures similar to those in $5 \mathrm{e}$ with the addition of neutralizing antibodies to IL-6 and IL-23. Data are the average \pm s.e.m. pooled from three independent experiments. ${ }^{*} P<0.01$, ${ }^{* \star} P<0.01$ (Student's unpaired $t$-test).

Previous studies have shown that CCL20 production and recruitment of CCR6 + DC occur during infection in mucosal sites, such as the gut ${ }^{20,41,42}$ and lung. ${ }^{43}$ This migration and activation of mucosal DC has also been shown to be an important host strategy for the induction of pathogen-specific effector T-cell responses. ${ }^{18,20}$ Previous studies have illustrated that the induction of CCL20 and migration of CCR6 + DC can occur in a TLR5-dependent manner, stimulated by flagellins present on invading bacteria. ${ }^{11,29}$ Our study does not contradict a role for TLR5 in CCL20 production. In fact, Caco-2 cells, which express TLR $5^{11}$ but not TLR $1^{28}$ (data not shown), were able to produce CCL20 when stimulated with Y. enterocolitica. Notably, when wild-type human TLR1 (602I) was transfected into the Caco-2 cells, there was an increase in CCL20, indicating that these two TLR receptors may synergize to promote higher
CCL20 levels. Interestingly, when TLR1 contained a SNP that prevents cell surface expression, there was no change in the levels of CCL20. This SNP is particularly interesting because it has been associated with mucosal disease such as IBD. ${ }^{44}$ However, the effect that TLR1 deficiency has on mucosal immunity has never been fully examined. These data suggest that the absence of TLR1 signaling may result in aberrant DC activation and a defect in antigen-specific priming of effector $\mathrm{T}$ cells. How these defects may contribute to autoimmunity and infection is currently under investigation.

Although TLR2/1 signaling in DC has been shown to activate a gut-specific phenotype,${ }^{45}$ very little is known about the role of TLR1 activation in the intestinal epithelium of the small intestine. The present study demonstrates a profound defect in CCL20 production from the intestinal epithelium in the 
absence of TLR1 signaling. Although this is the first study that defines a role for TLR1 in the intestinal epithelium, studies have defined a role for its co-receptor, TLR2, in mucosal immunity. TLR2 signaling has been shown to be important for mucosal repair ${ }^{46}$ and regulation of intestinal barrier function. ${ }^{47}$ However, these studies were performed on mice with deletion of the TLR2 gene. Consequently, heterodimers with either TLR6 or TLR1 would be unable to form, thus impacting signaling via all three receptors. Here, we show a protective role for TLR1 during bacterial infection via its signaling in the intestinal epithelium. Taken together with our previous work, we show that TLR1 can have multiple, non-redundant roles depending upon the tissue or the type of cell on which it is expressed. TLR1 signaling in the epithelium induces CCL20 and recruits DC important for generating anti-Yersinia $\mathrm{T}_{\mathrm{H}} 17$ cells. However, TLR1 signaling in MLN DC produce IL-6 and IL-23, but not CCL20. These data confirm that the context and tissue in which the pathogen is detected ultimately determines the type of immune response induced.

This study defines TLR1 as a critical receptor involved in the initiation of immune response via migration and activation of DC in the gut-associated lymphoid tissue and ultimate clearance of mucosal pathogens through the induction of effector $\mathrm{T}_{\mathrm{H}} 17$ cells. The early events of an immune response are critical for the control and containment of the pathogen, as well as recruiting professional antigen-presenting cells to the site of infection. During mucosal infection, pathogens will interact with the intestinal epithelium, so the ability of these cells to communicate with the immune system and signal to DC is critical for the generation of anti-bacterial or anti-viral T-cell responses. Here, we demonstrate that TLR1 signaling in the epithelium induces the production of CCL20. The production of CCL20 recruits DC that function to phagocytose $Y$. enterocolitica and induce anti-Yersinia $\mathrm{T}_{\mathrm{H}} 17$ cells. Thus, TLR1-signaling links the innate responses and the adaptive response via the recruitment and activation of CCR6 + DC. Although the data presented here demonstrate that TLR1 activates DCs during pathogenic infection, there remains a possibility that TLR1 could also trigger DC activation against commensal microbiota if the mucosal barrier is damaged during inflammation. This is especially intriguing, as both CCL20 and CCR6 have been identified as susceptibility genes in Genome Wide Association Studies for IBD. ${ }^{22}$ However, the molecular mechanism for the induction of CCL20 during IBD has not been clearly defined.

In summary, our study expands upon our understanding of how TLR1 may induce mucosal $\mathrm{T}_{\mathrm{H}} 17$ responses and identifies a novel role for TLR1 at the intestinal epithelium via induction of CCL20 and activation of mucosal DC. These data are important as they suggest that stimulation of TLR1 during vaccination or infection would promote immunity, generation of pathogenspecific $\mathrm{T}$ cells, antibody production, and eventual clearance of the bacteria. Alternatively, inhibition of TLR1 may dampen chronic inflammation by inhibiting the generation of antigenspecific $\mathrm{T}_{\mathrm{H}} 17$ cells, which may provide a therapy for patients with mucosal autoimmune disease or IBD.

\section{METHODS}

Mice. TLR $1^{-1-}$ mice were generously provided by S. Akira and then bred to C57BL/6 mice. Wild-type littermates were used as controls, unless otherwise noted in figure legends. All mice were maintained at The University of Southern California and all animal experiments were performed in accordance with institutional guidelines.

Bone marrow chimera's. TLR $1^{-1-}$ and wild-type littermate control mice were lethally irradiated with $1,000 \mathrm{cGy}$. The mice were reconstituted intravenously with $10 \times 10^{6}$ cells isolated from donor bone marrow. After 3 weeks, the mice were infected orally with $1 \times 10^{6}$ c.f.u. Y. enterocolitica. At the time of collection, isolated LPCs and IECs were assayed for expression of TLR1 to confirm the reconstitution.

Bacterial growth, culture and infections. Y. enterocolitica strain 8081 and various mutants $\Delta l c r V, \Delta y s c, \Delta i n v A$ were grown overnight at $26^{\circ} \mathrm{C}$ in tryptic soy broth (BD Biosciences, San Jose, CA). Following overnight incubation, each strain was subcultured by a 1:100 dilution into fresh growth medium and further incubated for $2 \mathrm{~h}$. Bacterial density was measured by OD at $600 \mathrm{~nm}$ and diluted to $1 \times 10^{6}$ c.f.u. per $\mathrm{ml}$ in sterile phosphate-buffered saline (PBS). Mice were administered $100 \mu \mathrm{l}$ by intragastric gavage. Custom-made goat anti-murine CCL20 was prepared by Invitrogen Life Technologies (Grand Island, NY) by multiple site immunization of a goat with recombinant mouse CCL20 (R\&D System, Minneapolis, MN) emulsified in Complete Freund's Adjuvant. Antiserum was titrated by sandwich ELISA and specificity was verified by the failure to cross-react with any other chemokine tested (e.g., mouse CXCL10, CXCL12, CCL5, CCL3, CXCL13, CCL2, CCL21, and CCL22). In some experiments, mice were treated intraperitoneally with polyclonal anti-CCL20, and as a control goat $\operatorname{IgG}(\mathrm{R} \& \mathrm{D}$ Systems), beginning at the time of infection and then every other day for 5 days. Bacterial burden was determined following the collection of MLN, which were homogenized in sterile PBS. Samples were then serially diluted in sterile PBS, plated onto Trypic Soy Agar plates (BD Biosciences) and grown for $48 \mathrm{~h}$ at $26^{\circ} \mathrm{C}$.

Yersinia-specific Antibody ELISA. Ninety-six-well plates were coated with $10 \mu \mathrm{g} \mathrm{ml}^{-1} Y$. enterocolitica lysate overnight at $4{ }^{\circ} \mathrm{C}$ prepared as previously described. ${ }^{36}$ Briefly, strain 8081 was grown as described above. The bacteria was pelleted and reconstituted in $0.5 \mathrm{ml}$ sterile PBS. Silicon beads were added to the bacteria and the solution was lysed using a bead beater (Biospec, Bartlesville, OK) for $5 \mathrm{~min}$. Protein determination was performed on the lysate and $100 \mu$ lysate was plated to confirm all the bacteria were killed. Fecal samples were prepared by collecting fresh fecal pellets that were dissolved in $1 \mathrm{ml}$ lysis buffer containing soybean trypsin. Undiluted fecal supernatants were added to bacteria-coated plates for $2 \mathrm{~h}$ at room temperature. Plates were washed and then incubated with HRP-conjugated anti-mouse IgA (Santa Cruz Biotechnology, Santa Cruz, CA), rabbit anti-mouse IgG1, rabbit anti-mouse IgG2a, or rabbit anti-mouse IgM followed by anti-Rabbit HRP (BD Biosciences). 3,3'-5,5'-tetramethylbenzidine substrate (Dako, Carpinteria, CA) was used for detection and absorbance was read at $495 \mathrm{~nm}$ OD. Antibody titers were detected by ELISA and calculated according to the formula: $\left(\mathrm{OD}_{450 \mathrm{~nm}}\right.$ sample $-\mathrm{OD}_{450 \mathrm{~nm}}$ of blank) $\times$ serum dilution, where OD is optical density.

Detection of cytokines. Isolated IEC, LP, and MLN cells were collected and prepared for ELISA as previously described. ${ }^{48}$ Quantification of CCL20 by ELISA (R\&D Systems) was performed by manufacturers specifications. For the detection of IL-17 in response to Yersinia, LP cells were isolated as previously described ${ }^{48}$ and the cells were stimulated with $10 \mathrm{mg} \mathrm{ml}^{-1}$ Y. enterocolitica lysate prepared as described. ${ }^{23}$ The supernatants were collected after $24 \mathrm{~h}$ and IL-17 was detected by ELISA (R\&D Systems).

Caco-2 transfections. Caco-2 cells (ATCC CRL-2102) were cotransfected with a vector expressing TLR1 I602I (wild type) or a SNP in TLR1 I602S (generously donated by Dr Richard Tapping, University of Illinois at Urbana-Champaign). This vector also expresses the firefly 
luciferase gene driven by the IL-8 promoter and pRL-null, a Renilla luciferase transfection control (Promega, Madison, WI). Transfections were performed using FuGENE 6 at a lipid-to-DNA ratio of 4:1 (Roche Applied Science, Indianapolis, IN). The total amount of transfected plasmid DNA was equalized by supplementing with empty vector, pFLAG-CMV. Two days post transfection the cells were stimulated with the TLR1/2 agonist (Pam3CysK4) (Invivogen), TLR6/2 agonist (FSL-1; Invivogen), TLR4 agonist (LPS; Sigma), or Y. enterocolitica lysate for $18 \mathrm{~h}$, and cell lysates were collected. Firefly luciferase and Renilla luciferase enzyme activities were determined using the luciferase assay system (Promega, Madison, WI) according to the manufacturer's instructions. Firefly luciferase activity was normalized to that of Renilla luciferase activities to correct for the transfection efficiency. After correcting for transfection efficiency, all values were normalized to those of unstimulated cells transfected with reporters and empty FLAG-CMV vector.

Flow cytometry. PP cells $\left(0.5-1 \times 10^{6}\right)$ were washed and incubated with anti-mouse FcRII/III (CD16/32 clone 2.4G2; BD Biosciences) for $15 \mathrm{~min}$ at $4{ }^{\circ} \mathrm{C}$ to prevent nonspecific Fc binding. Cells were washed in PBS with 10\% BSA (Sigma Aldrich, St Louis, MO) and stained with a live/dead marker (7-AAD) before being labeled directly with monoclonal antibodies specific for lymphocyte surface markers (CD13 FITC, CD11c FITC, CD45 APC-Cy7, and CCR6 PE; BD Biosciences) at $4{ }^{\circ} \mathrm{C}$ for $15 \mathrm{~min}$. Expression was determined on a FACSCanto flow cytometer (BD Biosciences) using FloJo software.

DC and T cell co-culture. CCR6 + or CCR6-DCs were sorted from the PP of wild-type mice infected with Y. enterocolitica for 3 days. $5 \times 10^{4}$ DCs were co-cultured with $1 \times 10^{5}$ naive CD4 T cells isolated from OT2 mice in the presence or absence of $10 \mu \mathrm{g}$ ovalbumin for 3 days. IL17 levels were measured by ELISA. In some experiments, CD4 T cells were sorted from the co-culture using anti-CD4 microbeads (Miltenyi Biotech, Bergish Gladbach, Germany) and mRNA levels of rorgt were measured by quantitative RT-PCR or the addition of $1 \mu \mathrm{g} \mathrm{ml}^{-1}$ antiIL-6 (R\&D Systems) and anti-IL-23 (R\&D Systems) were added to the cultures.

Quantitative RT-PCR. Tissue RNA was isolated from Peyer's patch using TRIzol reagent (Life technologies). RNA was transcribed into cDNA using the iScript cDNA synthesis kit (Bio-Rad Laboratories, Irvine, CA). Quantitative real-time RT-PCR was performed on an iCycler iQ real-time PCR detection system (Bio-Rad Laboratories) using a SYBr green amplification kit (Bio-Rad Laboratories) with the following primers, $i l-6$ forward $5^{\prime}$-GCCCAGCTATGAACTCCTTCT$3^{\prime}$ and reverse 5'-GAAGGCAGCAGGCAACAC-3', il-23 forward $5^{\prime}$-GGTGGCTCAGGGAAATGT- $3^{\prime}$ and reverse $5^{\prime}$-GACAGAGCAG GCAGGTACAG-3' ${ }^{\prime}$, and $c c l 20$ forward $5^{\prime}$-AGATGGCCGATGAA GCTTGTGA-3'， ccl20 reverse $5^{\prime}$-TCATTTCCTCCTTGGGCTGT GT- $3^{\prime}$, rorgt forward $5^{\prime}$-ACAGCCACTGCATTCCCAGTTT- $3^{\prime}$ and rorgt reverse 5'-TCTCGGAAGGACTTGCAGACAT-3'. Gene expression levels for each individual sample were normalized to GAPDH. Fold changes in gene expression were relative to uninfected controls and calculated using the $\Delta \Delta C t$ method.

Derivation of human CD34 + DC. CD34 ${ }^{+}$cells were isolated from mononuclear fractions of normal human blood purchased from Bioreclamation (Jericho, NY) using positive selection with anti-CD34coated microbeads (Miltenyi Biotec) to a purity of $89-95 \%$. Cells were cultured in complete medium containing RPMI 1640 (Invitrogen, Breda, The Netherlands) supplemented with $8 \%$ heat-inactivated fetal calf serum (FCS) (Hyclone; Thermo Fisher Scientific, Rockford, IL), $10 \mathrm{~mm}$ HEPES (Invitrogen), $2 \mathrm{~mm}$ L-glutamine (Invitrogen), $50 \mu \mathrm{M}$ $\beta$-mercaptoethanol, and penicillin/streptomycin (Invitrogen). From day 0 to day 6 , the cells were cultured in complete medium supplemented with $100 \mathrm{ng} \mathrm{ml}^{-1}$ granulocyte macrophage colonystimulating factor ( $R \& D$ systems) and $5 \%$ heat inactivated $\mathrm{AB}^{+}$ pooled human serum (Gemini Bio-Products West Sacramento, CA).
On the final day, the cells were counted and used in the chemotaxis assay described below.

Chemotaxis Assay. Chemotaxis was performed in triplicate for $2 \mathrm{~h}$ in Boyden chambers (Neuroprobe, Gaithersburg, MD). Supernatants from Caco-2 cells stimulated with $Y$. enterocolitica, $10 \mu \mathrm{g} \mathrm{ml}^{-1}$ rhCCL20 (positive control; R\&D Systems), or media alone (negative control) were added to the top chamber. In all cases, anti-CCL20 $\left(1 \mu \mathrm{g} \mathrm{ml}^{-1} ; \mathrm{R} \& \mathrm{D}\right.$ Systems) antibody was added to ensure specificity of migration. A total of $5 \times 10^{5}$ human-derived CD $34+$ DCs were added to the lower wells. After $2 \mathrm{~h}$, the top chambers were harvested and enumerated.

Statistics. Paired and unpaired Student's $t$-tests were used when noted in figure legends. Tests that had an interaction of $P<0.05$ were considered significant. Wilcoxon log-rank test was used for analysis of mouse survival in animal challenge experiments.

\section{ACKNOWLEDGEMENTS}

This work was supported by NIH (DK-82725-2) (RWD).

\section{DISCLOSURE}

The authors declare no conflict of interest.

c) 2013 Society for Mucosal Immunology

\section{REFERENCES}

1. Hooper, L.V. \& Macpherson, A.J. Immune adaptations that maintain homeostasis with the intestinal microbiota. Nat. Rev. Immunol. 10, 159-169 (2010).

2. Galan, J.E. \& Curtiss, R. III Cloning and molecular characterization of genes whose products allow Salmonella typhimurium to penetrate tissue culture cells. Proc. Natl. Acad. Sci. USA 86, 6383-6387 (1989).

3. Khoramian-Falsafi, T., Harayama, S., Kutsukake, K. \& Pechere, J.C. Effect of motility and chemotaxis on the invasion of Salmonella typhimurium into HeLa cells. Microb. Pathog. 9, 47-53 (1990).

4. Grassl, G.A., Bohn, E., Muller, Y., Buhler, O.T. \& Autenrieth, I.B. Interaction of Yersinia enterocolitica with epithelial cells: invasin beyond invasion. Int. J. Med. Microbiol. 293, 41-54 (2003).

5. Iwasaki, A. \& Medzhitov, R. Toll-like receptor control of the adaptive immune responses. Nat. Immunol. 5, 987-995 (2004).

6. Coombes, J.L. et al. A functionally specialized population of mucosal CD103 + DCs induces Foxp3 + regulatory T cells via a TGF-beta and retinoic acid-dependent mechanism. J. Exp. Med. 204, 1757-1764 (2007).

7. Matzinger, P. \& Kamala, T. Tissue-based class control: the other side of tolerance. Nat. Rev. Immunol. 11, 221-230 (2011).

8. Sozzani, S., Allavena, P., Vecchi, A. \& Mantovani, A. The role of chemokines in the regulation of dendritic cell trafficking. J. Leukoc. Biol. 66, 1-9 (1999).

9. Ito, T., Carson, W.F.t., Cavassani, K.A., Connett, J.M. \& Kunkel, S.L. CCR6 as a mediator of immunity in the lung and gut. Exp. Cell Res. 317, 613-619 (2011).

10. Izadpanah, A., Dwinell, M.B., Eckmann, L., Varki, N.M. \& Kagnoff, M.F. Regulated MIP-3alpha/CCL20 production by human intestinal epithelium: mechanism for modulating mucosal immunity. Am. J. Physiol. Gastrointest. Liver Physiol. 280, G710-G719 (2001).

11. Sierro, F. et al. Flagellin stimulation of intestinal epithelial cells triggers CCL20-mediated migration of dendritic cells. Proc. Natl. Acad. Sci. USA 98, 13722-13727 (2001).

12. Gewirtz, A.T., Navas, T.A., Lyons, S., Godowski, P.J. \& Madara, J.L. Cutting edge: bacterial flagellin activates basolaterally expressed TLR5 to induce epithelial proinflammatory gene expression. J. Immunol. 167, 1882-1885 (2001).

13. Baba, M. et al. Identification of CCR6, the specific receptor for a novel lymphocyte-directed CC chemokine LARC. J. Biol. Chem. 272, 14893-14898 (1997).

14. Varona, R. et al. Molecular cloning, functional characterization and mRNA expression analysis of the murine chemokine receptor CCR6 and its specific ligand MIP-3alpha. FEBS Lett. 440, 188-194 (1998). 
15. Liao, F. et al. CC-chemokine receptor 6 is expressed on diverse memory subsets of $T$ cells and determines responsiveness to macrophage inflammatory protein 3 alpha. J. Immunol. 162, 186-194 (1999).

16. Schutyser, E., Struyf, S. \& Van Damme, J. The CC chemokine CCL20 and its receptor CCR6. Cytokine Growth Factor Rev. 14, 409-426 (2003).

17. Greaves, D.R. et al. CCR6, a CC chemokine receptor that interacts with macrophage inflammatory protein 3alpha and is highly expressed in human dendritic cells. J. Exp. Med. 186, 837-844 (1997).

18. Kallal, L.E., Schaller, M.A., Lindell, D.M., Lira, S.A. \& Lukacs, N.W. CCL2O/ CCR6 blockade enhances immunity to RSV by impairing recruitment of DC. Eur. J. Immunol. 40, 1042-1052 (2010).

19. Wu, Y.Y. et al. Upregulation of CCL20 and recruitment of CCR6 + gastric infiltrating lymphocytes in Helicobacter pylori gastritis. Infect. Immun. 75, 4357-4363 (2007).

20. Salazar-Gonzalez, R.M. et al. CCR6-mediated dendritic cell activation of pathogen-specific Tcells in Peyer's patches. Immunity 24, 623-632 (2006).

21. Kaser, A. et al. Increased expression of CCL20 in human inflammatory bowel disease. J. Clin. Immunol. 24, 74-85 (2004).

22. Barrett, J.C. et al. Genome-wide association defines more than 30 distinct susceptibility loci for Crohn's disease. Nat. Genet. 40, 955-962 (2008).

23. Depaolo, R.W. et al. A specific role for TLR1 in protective TH17 immunity during mucosal infection. J. Exp. Med. 209, 1437-1444 (2012).

24. Kang, J.Y. et al. Recognition of lipopeptide patterns by Toll-like receptor 2 Toll-like receptor 6 heterodimer. Immunity 31, 873-884 (2009).

25. Omueti, K.O., Beyer, J.M., Johnson, C.M., Lyle, E.A. \& Tapping, R.I. Domain exchange between human toll-like receptors 1 and 6 reveals a region required for lipopeptide discrimination. J. Biol. Chem. 280, 36616-36625 (2005).

26. Johnson, C.M. et al. Cutting edge: A common polymorphism impairs cell surface trafficking and functional responses of TLR1 but protects against leprosy. J. Immunol. 178, 7520-7524 (2007).

27. Wong, S.H. et al. Leprosy and the adaptation of human toll-like receptor 1. PLoS Pathog. 6, e1000979 (2010).

28. Melmed, G. et al. Human intestinal epithelial cells are broadly unresponsive to Toll-like receptor 2-dependent bacterial ligands: implications for hostmicrobial interactions in the gut. J. Immunol. 170, 1406-1415 (2003).

29. Rhee, S.H., Keates, A.C., Moyer, M.P. \& Pothoulakis, C. MEK is a key modulator for TLR5-induced interleukin-8 and MIP3alpha gene expression in non-transformed human colonic epithelial cells. J. Biol. Chem. 279, 25179-25188 (2004).

30. Kingma, S.D. et al. Lactobacillus johnsonii N6.2 stimulates the innate immune response through Toll-like receptor 9 in Caco-2 cells and increases intestinal crypt Paneth cell number in biobreeding diabetesprone rats. J. Nutr. 141, 1023-1028 (2011).

31. Shang, L. et al. Toll-like receptor signaling in small intestinal epithelium promotes B-cell recruitment and IgA production in lamina propria. Gastroenterology 135, 529-538 (2008).

32. Cornelis, G. R. The Yersinia Ysc-Yop 'type IIl' weaponry. Nat. Rev. Mol. Cell. Biol. 3, 742-752 (2002).

33. Matsumoto, H. \& Young, G.M. Translocated effectors of Yersinia. Curr. Opin. Microbiol. 12, 94-100 (2009).
34. Viboud, G.I. \& Bliska, J.B. Yersinia outer proteins: role in modulation of host cell signaling responses and pathogenesis. Annu. Rev. Microbiol. 59, 69-89 (2005).

35. Brodsky, I.E. et al. A Yersinia effector protein promotes virulence by preventing inflammasome recognition of the type III secretion system. Cell Host Microbe 7, 376-387 (2010).

36. Depaolo, R.W. et al. Toll-like receptor 6 drives differentiation of tolerogenic dendritic cells and contributes to LcrV-mediated plague pathogenesis. Cell Host Microbe 4, 350-361 (2008).

37. Mueller, C.A. et al. The V-antigen of Yersinia forms a distinct structure at the tip of injectisome needles. Science 310, 674-676 (2005).

38. Sibartie, S. et al. Modulation of pathogen-induced CCL2O secretion from HT-29 human intestinal epithelial cells by commensal bacteria. BMC Immunol. 10, 54 (2009).

39. Jaffar, Z., Ferrini, M.E., Herritt, L.A. \& Roberts, K. Cutting edge: lung mucosal Th17-mediated responses induce polymeric lg receptor expression by the airway epithelium and elevate secretory IgA levels. J. Immunol. 182, 4507-4511 (2009).

40. Sansonetti, P.J. \& Phalipon, A. M cells as ports of entry for enteroinvasive pathogens: mechanisms of interaction, consequences for the disease process. Semin. Immunol. 11, 193-203 (1999).

41. Khan, M.A. et al. Flagellin-dependent and -independent inflammatory responses following infection by enteropathogenic Escherichia coli and Citrobacter rodentium. Infect. Immun. 76, 1410-1422 (2008).

42. Mellits, K.H. et al. Induction of a chemoattractant transcriptional response by a Campylobacter jejuni boiled cell extract in colonocytes. BMC Microbiol. 9, 28 (2009).

43. Park, S.J. et al. Neutropenia enhances lung dendritic cell recruitment in response to Aspergillus via a cytokine-to-chemokine amplification loop. J. Immunol. 185, 6190-6197 (2010).

44. Pierik, M. et al. Toll-like receptor-1, -2, and -6 polymorphisms influence disease extension in inflammatory bowel diseases. Inflamm. Bowel Dis. 12, 1-8 (2006).

45. Wang, S. et al. MyD88-dependent TLR1/2 signals educate dendritic cells with gut-specific imprinting properties. J. Immunol. 187, 141-150 (2011).

46. Podolsky, D.K., Gerken, G., Eyking, A. \& Cario, E. Colitis-associated variant of TLR2 causes impaired mucosal repair because of TFF3 deficiency. Gastroenterology 137, 209-220 (2009).

47. Cario, E., Gerken, G. \& Podolsky, D.K. Toll-like receptor 2 enhances ZO-1associated intestinal epithelial barrier integrity via protein kinase C. Gastroenterology 127, 224-238 (2004).

48. DePaolo, R.W. et al. Co-adjuvant effects of retinoic acid and IL-15 induce inflammatory immunity to dietary antigens. Nature 471, 220-224 (2011).

This work is licensed under the Creative Commons Attribution-NonCommercial-No Derivative Works 3.0 Unported License. To view a copy of this license, visit http:// creativecommons.org/licenses/by-nc-nd/3.0/ 